Emotion Review

\title{
Piecing together emotion: Sites and time-scales for social construction
}

\begin{tabular}{|r|l|}
\hline Journal: & Emotion Review \\
\hline Manuscript ID: & EMR-10-280.R1 \\
\hline Manuscript Type: & Original Manuscript \\
\hline Aute Submitted by the & n/a \\
\hline Complete List of Authors: & Parkinson, Brian; University of Oxford, Experimental Psychology \\
\hline Area/Discipline: & social < psychology, cultural < psychology \\
\hline Keywords: & culture, evolution, development, dynamic systems \\
\hline & $\begin{array}{l}\text { This paper catalogs social processes contributing to construction of } \\
\text { emotions across three time-scales covering: natural selection; } \\
\text { ontogenesis; and moment-by-moment transactions. During human } \\
\text { evolution, genetic and cultural influences operate interdependently, } \\
\text { not as separate forces working against each other. Further, leaving } \\
\text { infants environment-open serves adaptive purposes. During } \\
\text { ontogenesis, cultural socialization affects emotion development in } \\
\text { various ways, not all of which depend on internalization of cultural } \\
\text { meanings as emphasized in some earlier social constructionist } \\
\text { accounts. Construction also operates over the moment-by-moment } \\
\text { time-scale of real-time interpersonal interactions, as emotions } \\
\text { adjust to the constraints and affordances of immediate cultural } \\
\text { environments. Claims that emotions are constructed, and that } \\
\text { social processes contribute to their construction, are no longer } \\
\text { contestable. Now the project is to specify how these processes } \\
\text { operate and what materials they use. }\end{array}$ \\
\hline Abstracthes \\
\hline
\end{tabular}

\section{SCHOLARONE ${ }^{m}$ Manuscripts}




\title{
Running Head: PIECING TOGETHER EMOTION
}

\author{
Piecing Together Emotion: Sites and Time-scales for Social Construction
}

Brian Parkinson

Oxford University, UK

Correspondence to:

Brian Parkinson

Oxford University

Department of Experimental Psychology

South Parks Road

Oxford OX1 3UD, UK

Phone: +44(0)1865-271423

Fax: +44(0)1865-310447

Email: brian.parkinson@psy.ox.ac.uk 
Piecing together emotion 2

\begin{abstract}
This paper catalogs social processes contributing to construction of emotions across three time-scales covering: natural selection; ontogenesis; and moment-bymoment transactions. During human evolution, genetic and cultural influences operate interdependently, not as separate forces working against each other. Further, leaving infants environment-open serves adaptive purposes. During ontogenesis, cultural socialization affects emotion development in various ways, not all of which depend on internalization of cultural meanings as emphasized in some earlier social constructionist accounts. Construction also operates over the moment-by-moment time-scale of realtime interpersonal interactions, as emotions adjust to the constraints and affordances of immediate cultural environments. Claims that emotions are constructed, and that social processes contribute to their construction, are no longer contestable. Now the project is to specify how these processes operate and what materials they use.
\end{abstract}

KEYWORDS: culture, evolution, development, dynamic systems 
Piecing together emotion 3

\section{Piecing Together Emotion: Sites and Time-scales for Social Construction}

Social constructionist approaches arose within a specific cultural and historical context. They represented an important corrective to essentialist ideas of emotions as irreducible subjective conditions or intact packages of response components. They attempted to do justice to the multifarious flexibility of emotions as lived and enacted in practical settings, as cultural and historical objects as well as cognitive and physiological ones. So far, so good.

However, calling people social constructionists quickly became an accusation about denying any impact of biology or evolution, and assigning priority to certain kinds of sociocultural explanation regardless of their specific applicability to the phenomena in question. The constructionist thesis was mistakenly seen as implying that emotions were nothing but social constructions, whose only existence was as cultural myths or artifacts. In this paper, I want to leave this baggage behind and embark afresh and unencumbered on an exploration of the parts of the social world that contribute to the construction of emotions. Thus, the term "social construction" is used here to describe the set of processes whereby interpersonal, institutional, and cultural factors make their mark as emotions are put together over time. No absolutist claim that all emotions are fully constituted by these processes is intended (see also Averill, 1992; Griffiths, 1997).

Some social influences on emotion construction are direct and immediate, while others are more historical and remote. To impose structure on their variety, I address three different time scales, each more local and specific than the last. First, I consider how social factors affect the genetic basis for emotional processes over the course of phylogeny. Second, I narrow down to an ontogenetic time frame and discuss how social 
factors shape development of emotions as infants grow into toddlers and toddlers into older children. Finally, I zero in on moment-by-moment pushes and pulls exerted by the social world as emotions unfold in real-time transactions.

Social constructionist theories often explain emotions in terms of socialization processes operating within an ontogenetic time-frame (e.g., Saarni, 2001). Their central focus falls on the role of individual internalization of cultural meanings or scripts in determining emotional structure. In this paper, I want to argue that this particular kind of socialization is only one part of the story. Some aspects of the social construction of emotion operate in advance of ontogenesis (as products of cultural history, see Matt, 2011, as well as the phylogenetic influences emphasized in the present paper). Others come into play only when ontogenetically derived capacities are put into contact with real-time interpersonal and cultural affordances. Further, emotion socialization during ontogenesis is not simply a matter of internalizing culture. Culture is also part of the practical environment to which infants respond, and shapes caregiver responses to their nascent emotions before they have any apprehension of emotion's cultural significance.

\section{Socializing Evolution}

Everybody knows that emotions have phylogenetic histories. In the bad old days, evolution's relative explanatory importance was pit against influences operating over the more manageable time-scale of socialization. The more nurture, the less nature, and vice versa. Such a simplistic additive formula no longer makes sense for two reasons. On the one hand, gene operation is modulated by cultural factors. On the other, culture itself has a genetic component. The following sections develop each of these points in turn. 
Piecing together emotion 5

\section{Culture-dependent genetic effects}

The mechanics of genetic transmission and replication that permit natural selection are not driven solely by internal characteristics of inherited DNA sequences. Intimate interrelations and synergies between cultural and biological processes operate right down to the cellular level. For example, which particular nucleotide patterns are combined in reproduction depends on societal and institutional courtship practices, norms of attractiveness, and conventions about appropriate sexual partners. Thus selection is never purely "natural" among humans but always already cultural.

After fertilization, individual ("homunculoid") genes do not deliver preset commands that directly shape predefined mental and bodily structures (e.g., Oyama, 1985). Instead, they operate in interaction with one another in ways that are responsive to their metabolic milieu, first in and then outside the uterus (e.g., Meaney, 2010). And the actions and reactions of the bodies that carry the genes (including the mother's during interuterine development) are in turn attuned to cultural pressures and affordances. Whatever someone is doing in the social world is embodied in biochemical adjustments that may modulate DNA transcription and expression. To the extent that what they are doing depends on local culture, culture too penetrates right down to genetic effects. Processes of contextualized gene-guided development continue to operate as a baby grows older with vanishingly few developmental trajectories immune to social and cultural influences.

Recent animal research provides an illustration of how socialization practices might exert effects on the expression of genes relating to emotional behaviour. Rat pups whose mothers lick and groom them more (or who are exposed to experimental 
Piecing together emotion 6

manipulations of stroking) subsequently show lower levels of behavioral and hypothalamic-pituitary-adrenal (HPA) responses to stressful stimulation (e.g., Burton et al., 2007). The mechanism for this effect seems to relate to the regulation of corticotrophin releasing factor (CRF) in the hypothalamus by glucocortisoid receptors, whose expression relates directly to the extent of maternal licking and grooming (Francis et al., 1999). Thus, inter-organism interactions affect the production of transcription factors, thereby modifying the biochemical effects of DNA, with broader consequences for emotional development (see Meaney, 2010 for a more detailed account).

In humans, closeness of caregiver-infant contact and extent of mutual touch depend partly on cultural norms and practices (e.g., Keller et al., 2004; Richman et al., 1988). Although it would be premature to draw direct parallels with the animal research described above, the findings at least suggest plausible biological mechanisms permitting cultural effects operating even on low-level genetic processes. Further down the line, which genes get selected may in turn depend partly on the prevailing cultural environment that shapes their expression and consequently their specific effects on developing brains and bodies. In other words, the same gene may have different and differentially adaptive effects depending on the way that culture affects its expression. Genetically shaped socialization

In the previous section, I gave examples of ways in which socio-cultural factors might affect genetic processes that shape some of the raw materials required for emotion construction. One implication is that genetic and cultural influences cannot easily be separated even when considered at the micro-level. However, this interdependence applies to effects of genes on culture as well as effects of culture on genes. Not only does 
the operation of genetic mechanisms partly depend on cultural processes, but also these social processes themselves partly depend on genetic mechanisms (whose operation depends on cultural processes and so on). Socialization itself is a process that is influenced by natural selection (rather than a process working in opposition to it, e.g., Flinn \& Alexander, 1982; Greenfield, 2002). Humans are designed to need socializing and equipped to take advantage of it. Infants are innately neotenous and environmentopen for good adaptive reasons. In other words, culture is important to emotion socialization partly because inherited genes contribute to the capacity to benefit from its availability.

Turning from the receivers of socialization practices (infants and children) to their providers (caregivers), humans have a capacity (to learn over the course of development) to provide the kinds of caregiving that socialize, and to respond to the intellectual, practical, and emotional needs of their offspring (e.g., intuitive parenting, Papoušek \& Papoušek, 1987, warmth, MacDonald, 1992). Although culture determines many of the particular ways in which we care for and about our children, some of our broader parental sensitivities and motivations are influenced by genetic processes too.

Co-evolved capacities for giving and receiving nurturance operate within a broader system of interpersonal, intergroup and institutional relations that are also themselves partly a product of evolved potentials for sociality and system-building (e.g., Boyd \& Richerson, 2009; Dunbar, 1998). A human being is the kind of animal that can capitalize on the many advantages of collective life (Tomasello, 1999). Correspondingly, natural selection need not operate by innately pre-wiring programs to meet survival and reproductive needs since it can also draw on resources provided by the social world 
within which human ontogenesis is inevitably embedded (e.g., as part of developmental systems, Griffiths \& Gray, 1994; Oyama, 1985).

None of this implies that sociocultural ecologies are fixed or determining. Indeed, part of the payoff of development by socialization is that groups and societies can develop new strategies and habits for dealing with historically and geographically variable challenges (Boyd \& Richerson, 2009). Humans are built to be adjustable, and culture provides some of the means by which adjustability is achieved.

These considerations carry obvious implications for debates surrounding the existence of innately preprogrammed "basic" emotions. Even if certain action readiness modes increase the probability of survival or reproduction under certain circumstances, there may be disadvantages to specifying them genetically as fixed packages of response. Human infants' capacities for independently initiating survival-oriented actions are severely limited until a protracted period of socialization is well underway. Therefore, it may make more sense to exploit socialization processes to shape and structure modes of emotional response. This also brings the benefit of permitting adjustment to meet the needs of historically changing challenges and threats.

Correspondingly, the most adaptive actions that physically helpless infants could have in situations of direct survival threat are signals and appeals to caregivers that solicit their assistance. More generally, it serves obvious functional advantages to be equipped to maintain close relationships with caregivers and to be able to communicate important needs.

In sum, natural selection need not pre-specify fixed emotion programs but instead provides many of the materials out of which emotions are constructed over ontogenesis 
and in real-time transactions in the environment (Ortony \& Turner, 1990). These materials include social sensitivities that permit humans to participate in relationships and societies that will scaffold the structures that are assembled over the course of ontogenesis and during real-time adjustment to cultural ecologies. Further, the phylogenetic process itself has social aspects and involves co-evolutionary processes in which parental and cultural capacities complement emerging social orientations. In the next section, I discuss more directly how various social forces help to construct emotions out of the materials provided by evolution over the course of child development.

\section{Ontogenesis in Social Context}

As argued above, innate specification of emotions is limited (e.g., Bennett, Bendersky \& Lewis, 2002; Camras, 1992; Sroufe, 1996) for good adaptive reasons. Phylogeny subcontracts continuation of its construction work to ontogeny, partly because of ontogeny's better local knowledge and connections. Human babies start out with something (or a collection of somethings) that ultimately coalesces into loose patterns of relational orientations, activities and expressions that are taken as signs or symptoms of separable emotions. Cultural socialization of various kinds contributes to this coalescence. This section illustrates the numerous ways in which it might operate.

Let's start with minimal assumptions about innate infant predispositions. Babies have bodies and brains that take a certain general form, with limited degrees of freedom. They have sense organs that are attuned to certain regions of various spectra of environmental information (although their attunement is also shaped by early sensory experience, e.g., Hanganu-Opatz, 2010). Some sensations and stimuli make them feel 
better than others. They have a capacity to learn, and soon develop the ability to detect contingencies (Gergely \& Watson, 1999). They cycle between alertness and quiescence (e.g., Field, 1977). Intense and dynamic stimulus patterns attract their attention, especially during alert phases. Faces (and especially eye movements, Gliga \& Csibra, 2007) seem ideally configured for their sensitivities (Johnson \& Morton, 1991).

Otherwise, human infants start out relatively helpless and require care from others to survive. Indeed, they seem to be equipped with characteristics that tend to recruit such care (Papoušek \& Papoušek, 1987). It may not seem much, but couple even these basic characteristics with one or more older humans who provide dynamically contingent caring and stimulation, and time-structured relational patterns will coalesce. Embed all this in a specific ecological and cultural context and further fine-tuning is inevitable.

In the following subsections, I provide illustrations of some of the ways in which cultural and interpersonal processes contribute to the construction of emotion over the course of ontogenesis. First, I describe how culturally shaped caregiver responses shape developing infant expressions and emotions. Second, I consider how the calibration of emotional perspectives permitted by the onset of social referencing extends the realm of cultural influence. Finally, I say a few words about the contribution made by the subsequent internalization of cultural rules and meanings relating to emotion.

\section{Culturally shaped caregiver responsiveness}

Developmental psychologists have made importance advances in investigating emerging infant emotions in relation to their dynamic interpersonal context (e.g., Chow, Halligan, \& Messinger, 2010; Cohn \& Tronick, 1988; Fogel, 1993). Research shows that caregivers often mirror infant facial expressions in an exaggerated or stylized form 
(Holodynski \& Friedlmeier, 2006; Papoušek \& Papoušek, 1987), and orchestrate the dynamic structure of their interpersonal feedback to exploit infants' sensitivities (and to draw attention to certain temporal and configural aspects of unfolding affect, e.g., Stern et al., 1985).

Infants' adjustments to, and operations upon, the stream of hyper-responsive stimulation from caregivers soon consolidate into familiar cycles of co-regulated activity (e.g., Cohn \& Tronick, 1988). In particular, through reciprocal mirroring, infants come to display more differentiated and situationally-appropriate facial expressions, and to associate the facial configuration presented by their caregiver (and reflected in their own response) with their current relational activity (e.g., Gergely \& Watson, 1999;

Holodynski \& Friedlmeier, 2006).

Some aspects of early facial expression may (when taken in context) derive unambiguous universal meaning from their functional role in an ongoing transaction (e.g., gaze direction towards attractive objects or gaze aversion from repulsive ones, oral expulsion in response to unpalatable tastes). However, the specific patterns that are mirrored, emphasised or ignored by caregivers also depend on culturally learnt attunements and interpretations. For example, Holodynski and Friedlmeier (2006) argue that caregivers feed back conventionalised versions of facial expression that tune infants to local nonverbal dialects or accents (e.g., Elfenbein \& Ambady, 2002). In this and other ways, the early development of emotion expression may be channelled along cultural lines.

Culturally shaped parental reactions not only influence development of facial expression but also the relational functions of adopting particular modes of emotional 
engagement (Parkinson, Fischer \& Manstead, 2005). First, ethnotheories tend to attach greater importance to certain kinds of "hypercognized" emotions at the expense of others (“hypocognized" emotions, Levy, 1973). Caregivers subscribing to these ethnotheories inevitably attend more to (and regulate to a greater extent) infant emotions that are perceived to fall into the hypercognized category. Second, hypercognition is associated with cultural value, and greater attention is paid to emotions that are considered by society to be good or bad things to feel in particular contexts.

Caregiver responses to culturally valued and disapproved emotions differ in many ways. Attention may be withdrawn or punishment applied when a counternormative emotion seems to be present, whereas norm-compatible emotions are encouraged and elaborated. Thus, infants' emotional reactions are cultivated in ways that may bring them in line with societal prescriptions long before they internalise these prescriptions. For example, Lewis, Ramsay, and Kawakami (1993) found that Japanese infants aged between 2 and 6 months cried less than European-American infants of the same age when being given an injection, probably because of their early experiences of parental responses to crying in related situations.

The socio-cultural context for early emotion development includes not only socialized caregivers but also institutions and artifacts. These can help to reinforce and reproduce emotion norms by providing a specific ecology for parental and infant adjustment and interaction (see Parkinson, Fischer, \& Manstead, 2005). An everyday example is the use of the sucking device variously known as a "dummy" or "pacifier." This material object embodies and reproduces shared notions about causes and consequences of certain affective responses. Further, it carries implications about the 
legitimacy of particular forms of interpersonal regulation. Providing a pacifier not only changes current infant affect (e.g., Pinelli, Symington, \& Ciliska, 2002), but also sanctions and increases opportunities for certain emotion-regulation habits. Thus, infants given pacifiers may learn at an implicit level that repetitive movements of certain kinds can be comforting before they learn more explicit rules about when and how it is appropriate to comfort themselves using similar strategies.

\section{Social referencing}

Up to the age of around 1, most changes in infants' emotional life depend on the direct responsiveness of the material and social environment shaping infants' emotional orientations. A developmental milestone for toddlers involves learning to adjust their orientation to something as a consequence of another person's orientation: the phenomenon usually known as social referencing. For example, a one-year-old approaching a metre-deep drop covered with thick glass hesitates and looks across the precipice to his or her mother for guidance. If mother smiles back, the toddler is likely to crawl across, but if mother looks afraid or angry, the toddler is much more likely to stay put at the safe side of the visual cliff (Sorce et al., 1985).

Social referencing provides an additional, more flexible route whereby cultural values and norms may be transmitted from caregivers and others (e.g., Frith, 2008; Tomasello, 1999). Instead of responding directly to caregivers responses to them, toddlers now adjust to caregiver responses to objects in the shared environment. They learn that certain emotional orientations to certain objects are more culturally appropriate than others even when they are not in direct contact with those objects. They begin to develop emotional responses not just to people and objects but also to relations between 
people and between people and objects. To the extent that these relations are culturally shaped, the resulting emotions carry the imprint of the surrounding society (e.g., pride at team performance).

According to Holodynski and Friedlmeier (2006), a related development around this time is the capacity to recognise the symbolic, partly conventionalized, meaning of others' facial expressions, which emerges from caregivers' systematic reflection of stylized situationally appropriate faces as discussed above. Children start to pick up the contextual significance of facial expressions, permitting more sophisticated interpersonal emotion regulation. Ultimately, they also acquire the ability to use their own facial expressions symbolically and thereby influence others in complementary ways. Again, these developments require no internalization of explicit cultural norms or meanings but are nevertheless an important part of the cultural socialisation of emotional functioning.

\section{Representation and regulation}

It is often assumed that culture's effects on emotion construction mainly operate when children internalize local rules and meanings and apply them to emotional experience. Children come to represent their emotions in terms of authorized cultural categories, and regulate them according to prevailing rules, using available strategies.

This familiar social constructionist account of emotion socialization is certainly plausible and probably partly true. Learning ways of recognizing and distinguishing our own and others' emotions probably does transform our responses to them. Learning which of these culturally recognizable emotions are permissible in particular contexts probably does change our experience of them. And finding out how to downregulate culturally inappropriate emotions and upregulate culturally appropriate ones probably 
does make a difference to the way we (try to) deal with them. Being able to tell a child what to do and what not to do certainly makes a difference to the effectiveness of many forms of parenting, including those that bring effects on emotions.

However, each of these developments operates on capacities that have already been shaped by prior and more implicit forms of cultural socialization. Cultural differentiation of emotion begins when caregivers respond differentially to infant emotions. Cultural regulation begins when caregivers selectively attend to, reward or punish certain displays in particular contexts (and when they model regulation techniques such as attention diversion and active distraction). In all these cases, culture shapes emotions during early development because caregivers are culturally socialized and live with their offspring in culturally structured worlds. Subsequent internalization of rules and representations extends and reinforces functions that are already partly constructed by cultural meanings, rules, practices and ecologies (Parkinson, Fischer, \& Manstead, 2005).

\section{Shaping of Real-time Emotion}

It might seem that emotion construction reaches completion once a child is fully socialized. The (partly socially constructed) resources provided during phylogenesis (and cultural evolution) are assembled into differentiable response packages over the course of ontogenesis; end of story. Certainly, linguistically competent adults are able to represent their own and each other's experience in more or less consistent ways using differential emotion labels. But does this mean that the emotional realities so represented are 
themselves fully formed entities programmed into the mental system? Or is there still construction work to be done?

In the present section, I consider different ways in which interpersonal and cultural factors might make further contributions to emotion construction during real-time transactions with the practical and social environment. First, I consider the impact of moment-by-moment feedback in shaping the unfolding of executively controlled emotions. Second, I discuss how emotions might be assembled moment-by-moment from bottom-up processes even without top-down executive control (see also Parkinson, 2009).

Culturally shaped feedback

For many theorists (e.g., Cosmides \& Tooby, 2001; Ekman, 2003; Roseman, 2001), adult emotions are packaged responses co-ordinated by top-down executive processes initiated by appraisal. The individual's mental system detects a potential concern and activates a mode of action readiness designed (as a function of natural selection and/or socialisation) to deal with that concern. The emotion process is thus analogous to a kind of implicit decision-making that leads to a plan of action (e.g., Lazarus, 1991).

Because components are not tightly interlinked in most emotion episodes (e.g., Reisenzein, 2000), any executive program is unlikely to be fully fixed or deterministic. Instead, emotion syndromes probably follow principles of equifinality, in which alternative routes can lead to the same endpoint (see Campos, Dahl, \& He, 2010). In effect, emotion control operates at best as an open rather than closed system. Thus, the 
cognitive apparatus monitors the execution of the emotional agenda, making adjustments in response to internal and external feedback.

Some of this external feedback is shaped by cultural factors. In the previous section, I suggested that an emotion's cultural value determines caregiver encouragement or discouragement during emotion socialization. Similar processes are also likely to operate as adult emotions unfold moment-by-moment. In particular, other people may respond to socially disapproved emotions by withdrawal of social attention or active intervention of various kinds. These responses in turn are likely to bring effects on the emotion episode proceeds.

Correspondingly, others' responses to socially approved emotions may include a shared focus on the emotional object, and mirroring of expressive response, which may initiate social appraisal or contagion effects (e.g., Parkinson \& Simons, 2009). Having others around who share a common (or contrasting) orientation towards a current concern may make a difference to the direction and time-course of an unfolding emotion.

Culture also prescribes conventional practices for dealing with other people's emotions in real-time, such as ways of physically comforting someone who is upset (in certain circumstances), or providing reappraisals after minor setbacks ("it could have been worse"). More specific strategies are explicitly inculcated by certain organizations as part of training for emotional labor. For example, some flight attendants are told to treat angry passengers as if they were little children, or to adopt the role of a hostess welcoming guests to the flight deck as if it were a living room at home (Hochschild, 1983). These practices not only transform the emotions of workers who follow the 
prescribed feeling rules, but also those of clients whose affective responses adjust to the institutionally shaped interpersonal feedback.

\section{Emotions as emergent dynamic systems}

The previous section worked from the assumption that the internal organization of emotion syndromes partly derives from top-down executive control. Accordingly, realtime cultural influences on emotion construction socialization were restricted to feedback processes shaping the specific implementation of preset emotional agendas. However, it is also possible that some emotions coalesce in a more emergent manner as bottom-up processes interact over the course of ongoing transactions with the social and practical environment. If so, sociocultural factors may play a deeper role in the real-time construction of emotion syndromes.

For example, people may arrive at emotional orientations to ongoing events not because their appraisal systems have detected the need for an emotion in advance but merely as a function of the combined pressures and affordances of a dynamically structured evolving situation. Emotions may be situated actions rather than plans (Suchman, 1987, see also Parkinson, 2001b), and the processes guiding their course may be distributed between interactants, and between actors and the material and practical environment within which they operate (e.g., Griffiths \& Scarantino, 2009; Hutchins, 1995).

For example, my shouting may develop (moment-by-moment) into angry shouting when my voice raises progressively to overcome the ambient volume (as registered in your unreceptiveness, see Parkinson, 2001a). Correspondingly, your adjustment to my escalating emphaticness may itself turn into reciprocated anger 
(“there's no need to shout!"). At some point in this exchange, either or both of us might pick up the appraisals or emotions implied by our respective modes of engagement, but these individual apprehensions of meanings are not the main driving force behind the patterned co-regulated episode (Parkinson, 2009).

These possibilities are consistent with the principles of dynamic systems approaches (e.g., Fogel, 1993), which attribute emotion-structuring to influences operating separately on lower-level components (e.g., Ortony \& Turner, 1991) and the mutual associations and interdependencies that develop between them (see also Parkinson, 2009). In short, part of the coherence of many differentiated emotional events may derive from the interplay of culturally socialized agents operating within culturally structured ecologies. Socialization leaves its traces on these episodes in the form of perceptual attunements and reward sensitivities rather than by any internalisation of explicit rules or norms.

But do these instances of co-constructed mutual adjustment constitute bona fide cases of "emotion"? Not if a defining criterion is the individual apprehension of personal significance (e.g., Lazarus, 1984), the recognition (at some level) that the self is experiencing a particular emotion (e.g., Barrett, 2006), or the presence of an individual mental process that does the work of co-ordination (e.g., Scherer, 1993). Nevertheless, each interactant's mode of engagement conveys evaluation, is directed at a particular intentional object, and embodies a relational meaning. Further, there are continuities between the dynamics of these episodes and those that are perceived as less contestable emotions. In particular, co-constructed "emotions" may seamlessly transition into individually apprehended emotions in real-time transactions. On the broader time scale 
of ontogenesis, "emotions" operating as interpersonal action regulators may form the basis for the subsequent development of internalised control programs that output appraisal-based emotions.

Whatever we ultimately agree to call these patterns of co-regulated engagement, they bear close relations to uncontroversially emotional phenomena. Thus, their explanation is probably not wholly irrelevant to emotion psychology.

\section{Summary}

Many versions of social constructionism assume that emotions are assembled over the course of socialisation rather than as a result of natural selection. One problem with these accounts is that they tend to misrepresent or underestimate interdependences between phylogenesis and ontogenesis. A second limitation concerns the implicit assumption that emotions are pre-assembled constructions once socialisation is complete. The developmental trajectory is thought to reach its ultimate destination as soon as an adult is equipped with intact differential emotions, usually conceived as response packages or top-down control programs. But the capacity to act in culturally meaningful and/or biologically adaptive emotional ways need not depend on internal programming or top-down co-ordination. Instead, emotional structure may emerge as consequence of bottom-up interrelations between elements over the course of ontogenesis or from reciprocal adjustments to interpersonal behaviour and/or culturally structured environments. Much as evolution proceeds by niche-based selection of fortuitously adaptive mutations rather than by any divinely guided design process, socialisation and 
real-time responsive environments cumulatively shape emerging emotional modes of engagement instead of solely providing material for integration by internal representational and regulatory mechanisms.

\section{Conclusion and Future Directions}

This paper has set out a number of ways in which social processes are implicated in the construction of emotions as response syndromes or as more active modes of engagement with the practical and social environment. I have argued in particular that:

1. The genetic foundations for emotion are shaped by cultural and social forces from the outset, and leave room for further specification during socialization (for good adaptive reasons).

2. Caregivers' provision of structured nonverbal feedback shapes infants' nonverbal adjustments leading over time to culturally channeled expressive accents or dialects (e.g., Holodynski \& Friedlmeier, 2006).

3. Socialized norms and prescriptions about emotions affect caregiver responses to infants (including attentiveness, reward and punishment), and shape the development of their emotions long before infants internalize these norms and prescriptions explicitly.

4. Subsequently, social referencing permits toddlers to adjust their emotional orientations to the perceived orientation of other people, providing an additional and more flexible means of cultural influence. 
5. Articulated representations of emotions further extend infant capacities for emotion and emotion regulation but do not supersede or override earlier implicit competences.

6. Ontogenetic development equips children with sensitivities to certain kinds of feedback from ongoing transactions with the practical and social world that shape the emergence of real-time emotion without the necessity for top-down executive control.

7. Regulation of real-time emotion episodes may be distributed between interactants and between actors and environments.

The various processes described above are certainly both social and constructive in several senses. However, not all of them are easily accommodated within earlier social constructionist thinking about how cultural representations impose structure on emotion (e.g., Averill, 1980; Harré, 1986; Saarni, 2001). Despite the many links between them, the processes do not easily combine into a unitary theory of emotion. Indeed, the social aspects of many of the processes distinguished above operate as part of wider systems, and interpenetrate with constructed and reproduced material practices and artifacts (e.g., actor-networks, Latour, 2005). The emotional world is infused with sociality through and through, just as the social world is infused with emotionality.

Under these circumstances, it may be time to stop using the label "social constructionism" to describe a particular approach to emotion. Its implications of oneway cultural influence imposing structure on individual emotions can be restrictive, and its historical connotations can be misleading. Social constructionism as originally conceived has served its purposes, and the task now is to articulate more precisely the 
many ways in which various social processes are implicated in emotional life over the course of development and in real-time transactions. 


\section{References}

Averill, J. R. (1980). A constructivist view of emotion. In R. Plutchik \& H. Kellerman (Eds.), Theories of emotion (pp. 305-340). New York: Academic Press.

Averill, J. R. (1992). The structural bases of emotional behavior: A metatheoretical analysis. In M. S. Clark (Ed.), Review of personality and social psychology 13: Emotion (pp. 1-24). Newbury Park, CA: Sage.

Barrett, L. F. (2006). Solving the emotion paradox: Categorisation and the experience of emotion. Personality and Social Psychology Review, 10, 20-46.

Bennett, D. S., Bendersky, M., \& Lewis, M. (2002). Facial expressivity at 4 months: A context by expression analysis. Infancy, 3, 97-113.

Boyd, R. \& Richerson, P. J. (2009). Culture and the evolution of human cooperation. Philosophical Transactions of the Royal Society B. Biological Sciences, 364, $3281-3288$.

Burton, C. L., Chatterjee, D., Chatterjee-Chakraborty, M., Lovic, V., Grella, S. L., Steiner, M., \& Fleming, A. S. (2007). Prenatal restraint stress and motherless rearing disrupts expression of plasticity markers and stress-induced corticosterone release in adult female Sprague-Dawley rats. Brain Research, 1158, 28-38.

Campos, J. J., Dahl, A., \& He, M. (2010). Beyond breaches and battles: Clarifying important misconceptions about emotion. Emotion Review, 2, 100-104.

Camras, L.A. (1992). Expressive development and basic emotions. Cognition and Emotion, 6, 269-283.

Chow, S. M., Halligan, J. D., \& Messinger, D. S. (2010). Dynamic infant-parent coupling during the face-to-face/still-face. Emotion, 10, 101-114. 
Cohn, J. F., \& Tronick, E. Z. (1988). Mother-infant face-to-face interaction: Influence is bidirectional and unrelated to periodic cycles in either partner's behavior. Developmental Psychology, 24, 386-392.

Cosmides, L., \& Tooby, J. (2001). Evolutionary psychology and the emotions. In M. Lewis \& J.M. Haviland-Jones (Eds.), Handbook of emotions ( $2^{\text {nd }}$ edition, pp. 91115). New York: Guilford.

Dunbar, R. I. M. (1998). The social brain hypothesis. Evolutionary Anthropology, 6, 178190.

Ekman, P. (2003). Emotions revealed: Understanding faces and feelings. London: Weidenfeld \& Nicolson.

Elfenbein, H. A. \& Ambady, N. (2002). Is there an in-group advantage in emotion recognition? Psychological Bulletin, 128, 243-249.

Field, T. (1977). Effects of early separation, interactive deficits, and experimental manipulations on infant-mother face-to-face interaction. Child Development, 48 , 763-772.

Flinn. M.V., \& Alexander, R.D. (1982). Culture theory: The developing synthesis from biology. Human Ecology, 10, 383-400.

Fogel, A. (1993). Developing through relationships: Origins of communication, self, and culture. Chicago: University of Chicago Press.

Francis, D. D., Diorio, J., Liu, D., \& Meaney, M. J. (1999). Nongenomic transmission across generations in maternal behavior and stress responses in the rat. Science, $286,1155-1158$. 
Frith, C. D. (2008). Social cognition. Philosophical Transactions of the Royal Society B: Biological Sciences, 363, 2033-2039.

Gergely, G., \& Watson, J. S. (1999). Early socio-emotional development: Contingency perception and the social-biofeedback model. In P. Rochat (Ed.), Early social cognition: Understanding others in the first months of life (pp. 101-136). Mahwah, NJ: Lawrence Erlbaum Associates.

Gliga, T., \& Csibra, G. (2007). Seeing the face through the eyes: A developmental perspective on face expertise. Progress in Brain Research, 164, 323-339.

Greenfield, P. M. (2002). The mutual definition of culture and biology in development. In H. Keller, Y. H. Poortinga, \& A. Schoelmerich (Eds.), Between Culture and Biology (pp. 57-76). Cambridge: Cambridge University Press.

Griffiths, P.E. (1997). What emotions really are. Chicago: University of Chicago Press.

Griffiths, P. E., \& Gray, R. D. (1994). Developmental systems and evolutionary explanation. Journal of Philosophy, 91, 277-304.

Griffiths, P. E., \& Scarantino, A. (2009). Emotions in the wild: The situated perspective on emotions. In P. Robbins \& M. Aydede (Eds.), Cambridge handbook of situated cognition (pp. 437-453). Cambridge, United Kingdom: Cambridge University Press.

Hanganu-Opatz, I. L. (2010). Between molecules and experience: Role of early patterns of co-ordinated activity for the development of cortical maps and sensory abilities. Brian Research Reviews, 64,160-176.

Harré, R. (1986). (Ed.). The social construction of emotions. Oxford: Blackwell. 
Hochschild, A. R. (1983). The managed heart: Commercialization of human feeling. Berkeley, CA: University of California Press.

Holodynski, M., \& Friedlmeier, W. (2006). Development of emotions and emotion regulation. New York: Springer.

Hutchins, E. (1995). Cognition in the Wild. Cambridge, MA: MIT Press.

Johnson, M. H., \& Morton, J. (1991). Biology and cognitive development: The case of face recognition. Oxford: Blackwell.

Keller, H., Yovsi, R., Borke, J., Ka“rtner, J., Jensen, H., \& Papaligoura, Z. (2004).

Developmental consequences of early parenting experiences: Self-recognition and self-regulation in three cultural communities. Child Development, 75, 1745-1760.

Latour, B. (2005). Reassembling the social: An introduction to actor-network-theory Oxford: Oxford University Press.

Lazarus, R. S. (1984). On the primacy of cognition. American Psychologist, 39, 124-129.

Lazarus, R. S. (1991). Emotion and adaptation. New York: Oxford University Press.

Levy, R. (1973). Tahitians. Chicago: Chicago University Press.

Lewis, M., Ramsay, D. S., \& Kawakami, K. (1993). Differences between Japanese infants and Caucasian American infants in behavioural and cortisol response to inoculation. Child Development, 64, 1722-1731.

MacDonald, K. (1992). Warmth as a developmental construct: An evolutionary analysis. Child Development, 63, 753-773.

Matt, S. J. (2011). Current emotion research in history: Or, doing history from the inside out. Emotion Review, 3, 117-124. 
Meaney, M. J. (2010). Epigenetics and the biological meaning of gene-environment interactions. Child Development, 81, 47-79.

Ortony, A., \& Turner, T. J. (1990). What's basic about basic emotions? Psychological Review, 97, 315-331.

Oyama, S. (1985). The ontogeny of information: Developmental systems and evolution. Cambridge, UK: Cambridge University Press.

Papoušek, H., \& Papoušek, M. (1987). Intuitive parenting: A dialectic counterpart to the infant's integrative competence. In J. Orlofsky (Ed.), Handbook of infant development ( $2^{\text {nd }}$ ed., pp. 669-720). New York: Wiley

Parkinson, B. (2001a). Anger on and off the road. British Journal of Psychology, 92, $507-526$.

Parkinson, B. (2001b). Putting appraisal in context. In K. R. Scherer, A. Schorr, \& T. Johnstone (Eds), Appraisal processes in emotion: Theory, research, application (pp. 173-186). Oxford University Press.

Parkinson, B. (2009). What holds emotions together? Meaning and response coordination. Cognitive Systems Research, 10, 31-47.

Parkinson, B., Fischer, A., \& Manstead, A. S. R. (2005). Emotion in social relations: Cultural, group, and interpersonal processes. Philadelphia, PA: Psychology Press.

Parkinson, B., \& Simons, G. (2009). Affecting others: Social appraisal and emotion contagion in everyday decision-making. Personality and Social Psychology Bulletin, 35, 1071-1084. 
Pinelli, J., Symington, A., \& Ciliska, D. (2002) Nonnutritive sucking in high-risk infants: Benign intervention or legitimate therapy? Journal of Obstetric, Gynecologic, and Neonatal Nursing: JOGNN/NAACOG, 31, 582-591.

Reisenzein, R. (2000). Exploring the strength of association between the components of emotion syndromes: The case of surprise. Cognition and Emotion, 14, 1-38.

Richman, A. L., LeVine, R. A., New, R. G., Horrigan, G. A., Welles-Nystrom, B., \& LeVine, S. (1988). Maternal behavior to infants in five cultures. In R. A. LeVine, P. M. Miller, \& M. M.West (Eds.), Parental behaviour in diverse societies (pp. 81-96). San Francisco: Jossey-Bass.

Roseman, I. J. (2001). A model of appraisal in the emotion system. In K. R. Scherer, A. Schorr, \& T. Johnstone (Eds), Appraisal processes in emotion: Theory, research, application (pp. 68-91). Oxford University Press.

Saarni, C. (2001). The social context of emotional development. In M. Lewis \& J.M. Haviland-Jones (Eds.), Handbook of emotions (2 ${ }^{\text {nd }}$ edition, pp. 306-322). New York: Guilford.

Scherer, K. R. (1993). Neuroscience projections to current debates in emotion psychology, Cognition and Emotion, 7, 1-41.

Sorce, J. F., Emde, R. N., Campos, J., \& Klinnert, M. D. (1985). Maternal emotional signaling: Its effect on the visual cliff behavior of 1 year olds. Developmental Psychology, 21, 195-200.

Sroufe, L. A. (1996). Emotional development: The organization of emotional life in the early years. New York: Cambridge University Press. 
Stern, D. N., Hofer, L., Haft, W., \& Dore, J. (1985). Affect attunement: The sharing of feeling states between mother and infant by means of intermodal fluency. In T. N. Field \& N. Fox (Eds.), Social perception in infants. Norwood, NJ: Ablex.

Suchman, L. A. (1987). Plans and situated actions: The problem of human-machine communication. Cambridge: Cambridge University Press.

Tomasello, M. (1999). The human adaptation for culture. Annual Review of Anthropology, 28, 509-529. 
1

2

3

4

5

6

7

8

9

10

11

12

13

14

15

16

17

18

19

20

21

22

23

24

25

26

27

28

29

30

31

32

33

34

35

36

37

38

39

40

41

42

43

44

45

46

47

48

49

50

51

52

53

54

55

56

57

58

59

60
Author Note

Work on this article was conducted as part of project EROS (Emotion Regulation of Others and Self) and supported by the Economic and Social Research Council, UK. 